\title{
A PROPÓSITO DE LA DIVERSIDAD DE CAPACIDADES Y NECESIDADES
}

\author{
ABOUT DIVERSITY AND ITS NEEDS
}

Antonio Rodríguez Fuentes ${ }^{1}$.

1 Doctor en Ciencias de la Educación, profesor de la Universidad de Granada (España). Correo: arfuente@ugr.es. 


\section{Resumen}

No son solo palabras. Las palabras refieren a conceptos. Luego están ligadas al pensamiento. $\mathrm{Y}$ determinan la acción. Las palabras no siempre son neutras. A menudo, arrastran una carga ideológica, un sentido y hasta una intención. Por ello, se hace preciso analizar no solo el pensamiento y la acción, sino también su mención, a través de la terminología empleada. El avance, el cambio, el progreso vienen marcados por el pensamiento que se materializa en la acción, pero todo está mediatizado por la nomenclatura. Por ello, se presenta una dilucidación terminológica y conceptual en el terreno educativo, cuando se presenta algún conflicto que requiere de una solución. Es decir, cuando se presenta alguna disfunción en el proceso de enseñanza-aprendizaje, que ha de formularse como necesidad educativa especial, como preludio de su respuesta educativa.

Palabras clave: Diversidad, Dificultades de enseñanzaaprendizaje, Necesidades Educativas Especiales.
It's not just words. Words refer to concepts. Then they are linked to thought. And they determine the action. Words are not always neutral. Often they carry an ideological burden, a meaning and even an intention. Therefore, it is necessary to analyze not only thought and action, but also its mention, through the terminology used. The advance, the change, the progress are marked by the thought that materializes in the action, but everything is mediated by the nomenclature. Therefore, a terminological and conceptual elucidation is presented in the educational field, when there is a conflict that requires a solution. That is, when there is a dysfunction in the teaching-learning process, which must be formulated as a special educational need, as a prelude to their educational response.

\section{Keyword:}

Diversity, Teaching-learning difficulties, Special Educational Needs. 
La Pedagogía, como cualquier disciplina científica, precisa una terminología propia pertinente. Empero, la lluvia terminológica de antaño requiere de un paraguas disciplinar y su filtro legislativo. Ellos han de actuar de protectores solo permeables al paso selecto de la nomenclatura educativa, atendiendo a su semántica y pragmáticas idóneas. Es más, ha de afanarse en elaborar una terminología correcta, como se ha venido haciendo en los últimos años.

Tras la implantación de la filosofía socioeducativa inclusiva para los sistemas educativos y el progreso evolutivo del corpus de conocimientos epistemológicos de la Educación Especial, se ha culminado la consolidación conceptual que le es propia. Y con ella la superación de una terminología que, por un lado, no resultaba plenamente adecuada $\mathrm{y}$, además, arrastraba un legado ideológico y una suscripción legal contrarios al movimiento inclusivo.

Hay algunos de esos términos que no vienen al caso en la actualidad. Están del todo superados por su propio (des)uso, significado e historia. Es el caso de algunos como minusválido, retrasado, impedidos, anormal, subnormal, deficiente, ineducable, etc. Afortunadamente, no resultan frecuentes en la jerga pedagógica. Lamentablemente, sí en la sociedad en general.

Centrando la atención en otros conceptos más confusos, conviene resaltar la discapacidad para aprender, en general, en etéreo, en absoluto, o los trastornos del aprendizaje. Sinceramente, tiendo a pensar que su razón de ser es el despropósito de alguien que presenta discapacidad para enseñar o está trastornado para ello. $\mathrm{O}$, en todo caso, por corrección política y amplitud de perspectiva, discapacidad no del individuo sino del currículo y/o la escuela, incapaces de hacer aprender y contribuir barreras para el aprendizaje. Ha sido denominado tal fenómeno como dispedagogía.

La realidad es que el alumno supuestamente discapacitado para aprender de hecho aprende; solo que el docente no lo percibe y la escuela y sus currículos no lo valoran pues esperan otros aprendizajes. El escenario simula al fenómeno del abejorro, el cual no puede volar según queda demostrado científicamente, pero él no lo sabe, y pues lo hace.

Uno de los primeros alumnos sordos que aprendió a hablar, en la edad media, emitió como primeras palabras en público que él era sordo 
pero no mudo $\mathrm{y}$, de hecho, pudo aprender a hablar. $\mathrm{Y}$ ello a pesar de que lo hizo mediante un programa denominado "el arte de enseñar a hablar a sordomudos", otro concepto a todas luces incorrecto.

Ahora bien, aunque sean externos al terreno educativo, sí se admiten los términos discapacidad y trastorno, pero deben ser bien empleados. Discapacidad sensorial (visual y/o auditiva), motora e incluso intelectual, y trastornos de la conducta y del desarrollo. Mejor no referir otras discapacidades.

$Y$ algunos de ellos pueden tener su origen en una deficiencia genética, congénita, heredada o adquirida. Lo cual ha sido puesto de relieve en los estudios de neuropsicología, neurodesarrollo e incluso de neuroaprendizaje.

Llegado a este punto, conviene dilucidar que no toda deficiencia genera una discapacidad o trastorno. Pues un déficits orgánico, genético, etc. puede no afectar a la capacidad del que lo padece, y/o dado los avances actuales puede mitigarse sus efectos. De igual forma, no toda discapacidad proviene de una deficiencia, sino que puede deberse a algún efecto secundario o colateral de un tratamiento, actividad, traumatismo, disfuncionalidad, etc.

Son términos, pues, a considerar en la dotación de recursos y condiciones para la enseñanza, pero por la amplitud de cada uno de ellos no aportan mucho por sí solos al docente. De hecho, tradicionalmente, incluso se han mal considerado como etiquetas para definir a las personas que las padecen, por encima de sus propias posibilidades reales, lo que ha sido uno de los grandes despropósitos de los sistemas educativos, materializado en la segregación hiperespecializada en centros específicos.

Otros términos han gozado de más recorrido, incluso siguen circulando actualmente por los trayectos pedagógicos. Las dificultades en o para el aprendizaje (DA) gozan de cierta aceptación, de hecho, se ha consolidado el concepto de dificultades específica de aprendizaje (DEA), para englobar la dislexia, la disortografía y la discalculia. $Y$ ello a pesar de seguir mostrando deficiencias, la más evidente es que resulta negativo, puesto que resalta la dificultad y no la posibilidad o la solución aparejada a ella. $Y$ ello supone un refuerzo y 
consolidación de las etiquetas anteriores.

Pero, además del componente actitudinal, existen evidencias de su impertinencia. Se muestra impreciso e incompleto al enfatizar el aprendizaje propio del aprendiz (alumno), en exclusividad. De no ser porque cuando se plantea cualquier DA para cualquier docente que se precie de serlo se plantea una dificultad para enseñar (DE).

$\mathrm{O}$, es más, acaso la DA no es más que consecuencia y su razón de ser de la DE. En todo caso, si el aprendiz no culmina el aprendizaje por el sendero que enseña el maestro, entonces este debe de enseñar de la manera que aprende el alumno. $Y$ volvemos con ello al campo del neurodesarrollo y neuroaprendizaje, y añadimos el de la neuroenseñanza.

Y, entonces, aparecen los anteriores términos unidos, cuando acontecen en el escenario escolar: dificultades de enseñanza-aprendizaje (DE-A), como las dos caras de una misma moneda, como dos roles, más que complementarios, necesarios de un mismo acto.

Incluso es superado, nuevamente, por otro concepto más completo, disfunciones en el proceso didáctico, en donde confluyen los agentes y elementos didácticos potencialmente erráticos: profesorado, alumnado, contextos y currículum, y sus conexiones. Es un término que se ha mostrado apropiado, y entronca con las nuevas corrientes de la neurodidáctica, neuroeducación o neuropedagogía.

Como quiera que más que hallar la causa y advertir el fallo consiste en buscar la solución o, aún mejor, la prevención, han emergido otros conceptos nuevos, y más idóneos, como la diversidad. Ahora bien, se empleó, a menudo, de sustituir, innecesariamente, al de discapacidad, que queda en otro plano no solo cronológico sino disciplinar.

Aun así, no resulta peyorativo ni negativo, incluso se colorea con una gama de tintes $y$ tonalidades diferentes que lo hacen lucir de riqueza para docentes y compañeros: diversidad de ritmos, de intereses, de conocimientos previos, de conductas. $\mathrm{E}$, incluso, de capacidades, lo que ha dado lugar al concepto de capacidades diversas; nuevamente con pretensión de superación del de discapacidades. También de funcionamiento, lo que se recoge como diversidad funcional.

Y caben, además, ser empleados para combatir las tradicionales 
etiquetas del alumnado por razones de discapacidad. Si bien, en el momento en que aparecen estos conceptos las etiquetas debieran haber quedado superadas. De lo contrario, volvemos de nuevo a la incompetencia de profesionales de la enseñanza.

Nótese que diverso es todo $y$ somos todos, así como la atención a la diversidad, expresión hiperempleada, tan diversa como su concepto. Incluso la atención educativa, incluso la atención escolar, incluso la atención didáctica. Ello podría erigirse como crítica, que se supera con la asunción de la diversidad como riqueza y beneficio para todos (alumnos $y$ docentes), en perjuicio de concepciones peyorativas anteriores.

Se ha aceptado el término, aprobado en memorándums internacionales, desde el informe Warnock de 1978, de necesidades educativas especiales (NEE), que no apunta ni al docente ni al discente, sino al contexto donde ambos se encuentran presentes. $Y$ si hubiera que apuntar, que solo lo haga el docente como arco para lanzar al alumno como flecha, con el prisma de una metodología eficaz y un horizonte de objetivos y contenidos adecuados, en el marco de una diana de aprendizajes y desarrollos para cada alumno.

Además, se pasa, por fin, al plural, por la asunción de que las NEE son múltiples (gallego y Rodríguez, 2016):

- Unas necesidades pueden ser intrínsecas al alumno. Por causa de una dificultad o no (e.g.: de un talento o potencial que alimentar). Que, a su vez, derive de una discapacidad o trastorno o no (e.g.: de los intereses y conocimientos previos no apropiados). Y causada por una deficiencia o no (e.g.: de la personalidad $\mathrm{o}$ del entorno discapacitante).

- Otras necesidades pueden ser extrínsecas, y entonces no están vinculadas a lo ontológico (alumno) pero sí a lo epistémico (contenido), metodológico (docente) y ecológico (contextual). Aunque a menudo olvidadas, es preciso cubrir estas necesidades para mitigar, así, su efecto contraproducente en el desarrollo del individuo, para superar las barreras de la discapacidad, en palabras de Barton (2006), luchando contra el discapacitismo.

La realidad es compleja y pues las anteriores NEE intrínsecas y 
extrínsecas pueden presentare conjuntamente.

Cabe, añadir además, la clasificación de las NEE atendiendo al criterio de su duración o persistencia. Pueden encontrarse:

- Unas necesidades temporales, cuando pueden ser superadas por el propio alumno de forma autónoma o tras un periodo de intervención educativa-escolar o de otro tipo (evolución, terapia, tratamiento, intervención quirúrgica, etc.).

- Otras necesidades se presentan permanentes, por cuanto acompañarán, presumiblemente, al alumno durante su periplo escolar (e.g.: $\quad$ sobre modalidades comunicativas necesarias por colectivos).

Igual que en la taxonomía anterior, estas NEE temporales y permanentes podrían presentarse junto con las anteriores en un mismo estudiante, en distintas asignaturas o capacidades. Es más por el propio desarrollo del alumnado una NEE puede cambiar de temporal a permanente y viceversa.

Evidentemente, también se diferencian las NEE por su intensidad, a saber:
- Unas necesidades serán leves, pues se distancian de manera poco significativa del currículo ordinario, y por tanto requerirán una modificación poco significativa.

- Otras necesidades son intensas, cuando el distanciamiento con respecto a los alumnos de sus edad y al currículo ordinario es mayor, demandando una alteración significativa.

También podrían combinarse las NEE leves e intensas, presentándose unas en unas áreas y otras para otras, o unas en un momento y otras en otro momento.

Por último, no en importancia, cabe distinguir entre NEE por su centro de atención:

- Unas necesidades son de tipo curricular, puesto que se orientan hacia alguna o algunas asignaturas áreas curriculares. Es decir, cuando existe un desfase curricular o un adelanto curricular.

- Otras necesidades son extracurriculares o preparatorias para lo curricular, cuando refieren a habilidades de vida diaria, sociales, comunicativas, de lengua oral, enriqueciendo socio-cognitivo, habilidades instrumentales básicas 
(atención, percepción y memoria), etc.

También puede encontrarse las NEE curriculares y extracurriculares por separado y conjuntamente en un mismo alumno.

Por tanto, dado que se trata de un asunto complejo, requiere una explicación compleja, basada en una completa evaluación psicopedagógica. Y se contempla la interacción de todas las causas anteriores bajo un paradigma holístico.

Conviene advertir que la realidad puede ser otra, según manifiestan y demuestran algunos autores (García, 2016), que consideran que el concepto de NEE puede constituirse como un lastre para la inclusión educativa y que se entiende de forma muy reduccionista, tanto que aconseja su cambio. Quizás por ello, se han desmembrado nuevos términos, como necesidades educativas específicas, necesidades específicas de apoyo educativo, y necesidades educativas diversas.

Incluso han surgido otros, supuestamente más inclusivos, como el de barreras para el aprendizaje y la participación (físicas, sensoriales, estructurales, o curriculares). $Y$ es que los términos "aprendizaje" $y$ "participación" son imprescindibles. En efecto, es sabida la regla de las "tres P" de la educación inclusiva propuesta por Ainscow, Booth y Dyson (2006):

- Presencia, contraria a la segregación escolar.

- Participación, contraria a la marginación en aula y/o centro.

- Progreso, contrario al fracaso educativo.

Navegando entre el laberinto terminológico y conceptual, representado en la figura 1 , no debe olvidarse lo fundamental, que es responder a esas necesidades educativas especiales. Para lo que resulta previo una buena identificación, holística e individual, de las mismas (Salvador, 2001) mediante su evaluación psicopedagógica. 


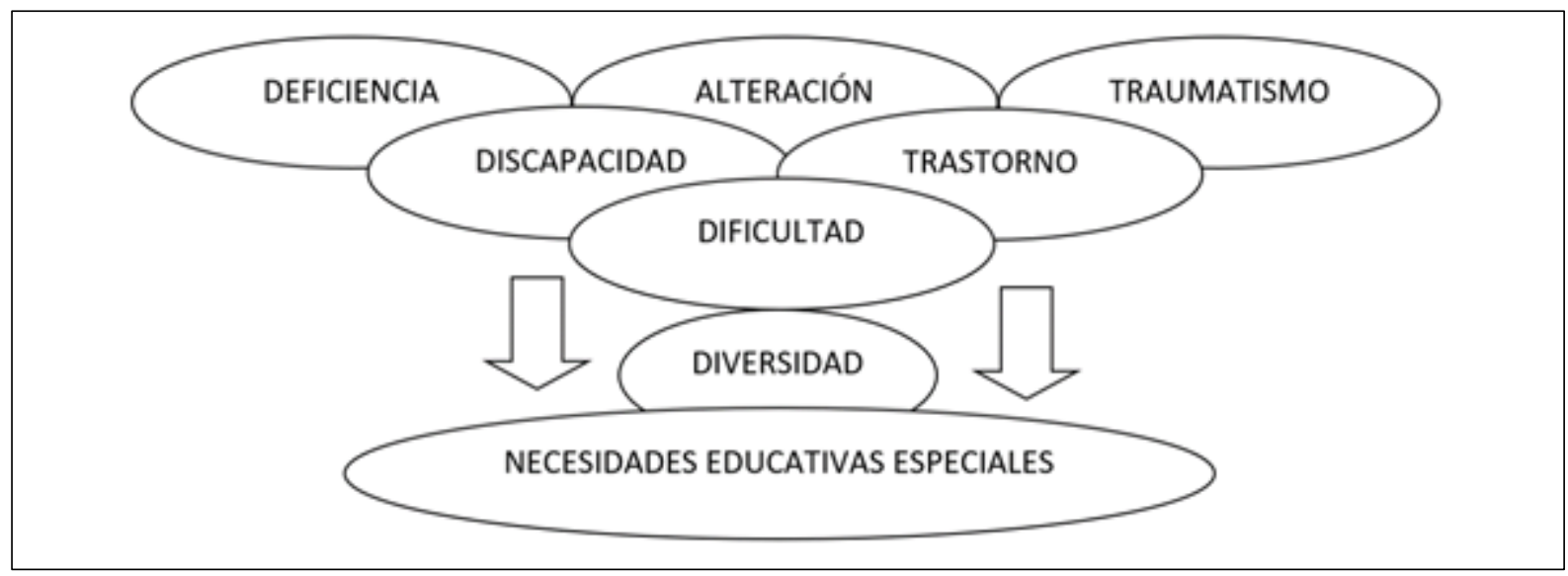

Figura 1. Terminología en el campo de la Educación Especial

Tal respuesta reclama el empleo de estrategias comunes para el conjunto del alumnado, y, en su caso, otras extraordinarias para determinados alumnos con nee. Se entra, entonces, en el plano de las adaptaciones, adecuaciones 0 modificaciones organizativas $\mathrm{y}$, sobre todo, curriculares (Rodríguez, 2015). Y en una modalidad de escuela inclusiva (Booth y Ainscow, 2011; Echeita, 2007), propuesta de ámbito internacional, que consolida también el concepto de NEE, en la Declaración de Salamanca de 1994 (UNESCO, 1994; Echeita y Verdugo, 2004).

$\mathrm{Si}$ bien, las anteriores adaptaciones curriculares (AC) no han quedado exentas, tampoco, de críticas por su falta de evidencias de eficacia. Desde un nuevo enfoque, más preventivo y proactivo, diferente al de las $A C$, que resultan de un prisma más correctiva y a posteriori, ha surgido, desde 1995 (CAST, 1995) el Diseño Universal de Aprendizaje (DUA).

La ventaja es la siguiente. Con la dinámica de las $A C$ se parte de un diseño curricular elaborado para un aluno prototipo o para la mayoría del grupo clase, el cual se va ajustando a una minoría de alumnado con NEE, lo que se antoja arduo $y$, a veces, inviable. EI DUA pretende atender a todos, incluidos las minorías, desde el principio, a priori, con propósito de minimizar o hacer innecesarias algunas modificaciones posteriores.

Puede concebirse como un enfoque basado en la investigación y derivado de la denominada "Pedagogía Accesible" (Rose et al., 
2014. p. 477), que también podría decirse inclusiva. De tal suerte, que se anticipe una programación curricular flexible acorde a las posibilidades y necesidades de cada alumno para el que se dirige, permitiendo las anteriores "tres P" a todos.

En todo caso, como constatan Simón et al. (2016, p. 15), "evidentemente ni las "AC" son el problema ni el "DUA" es la solución. Un análisis tan simplista estaría dejando fuera de nuevo la visión sistémica sobre la educación inclusiva". Se trata de combinar todo lo que tengamos a nuestro alcance, prevención y corrección, proacción y reacción, en definitiva, AC y DUA.

En este número aparecen apuestas diversas por recursos tecnológicos con una finalidad muy variopinta, que podrían formar parte de medidas de atención a la diversidad y respuestas a las NEE. Parten de capacidades y NEE de colectivos determinadas y se proponen como $\mathrm{AC}$ e, incluso, como DUA para optimizar el aprendizaje de todo el alumnado.

En primer lugar, se presenta una propuesta para contribuir a aumentar la calidad de la formación de profesionales en su educación superior, que proviene de Costa Rica.
Todo parte de la buena formación inicial docente, de ahí la preocupación loable por su calidad.

Otra propuesta versa sobre los recursos tecnológicos que existen al servicio de centros y familias en donde se precise del trabajo comunicativolingüístico de niños con Trastorno del Espectro Autista (TEA). Esta aportación proviene de España, y puede contribuir a configurar diseños universales para enseñar y aprender.

También desde España se recoge la propuesta de programa para trabajar las habilidades comunicativolingüísticas de alumnos con Trastorno Específico del Lenguaje (TEL). Los programas pueden erigirse como valiosas herramientas e instrumentos concretos para la intervención con alumnos con NEE. Pero requieren su personalización en la implementación para alumnos determinados, atendiendo precisamente a sus NEE

Y desde Panamá se ofrece un recurso tecnológico inédito para contribuir a la orientación y movilidad de personas con discapacidad visual. Contribuyen a la reducción de partida de las indicadas barreras para el aprendizaje y la participación, y por tanto, mejorando las posibilidades educativas del alumnado. 


\section{REFERENCIAS BIBLIOGRÁFICAS}

Ainscow, M., Booth, T. y Dyson, A. (2006). Improving Schools, Developing Inclusion. London: Routledge.

Barton, L. (2006). Overcoming Disabling Barriers. London: Routledge

Booth, T. y Ainscow, M. (2011). Index for Inclusion: developing learning and participation in schools (3rd ed.). Bristol: Centre for Studies in Inclusive Education (CSIE).

CAST (2008). Universal Design for Learning Guidelines. Versión 1.0. Wakefield, MA: Author.

Eceita, G. (2007). Educación para la inclusión o educación sin exclusiones. Madrid: Narcea.

Echeita, G. y Verdugo, M.A. (2004). La declaración de Salamanca sobre necesidades educativas especiales 10 años después. Valoración y prospectiva. Madrid: INICO.

Echeita, G., Sandoval, M. y Simón, C. (2016). Notas para una pedagogía inclusiva en las aulas. Actas IV Congreso Iberoamericano sobre el síndrome de Down. Salamanca.
Recuperado de: http://cddowninico.usal.es/docs/045.pdf

Gallego, J.L. y Rodríguez, A. (2016). La alteridad en educación. Teoría y práctica. Madrid: Pirámide.

García-Barrera, A. (2017). Las necesidades educativas especiales: un lastre conceptual para la inclusión educativa en España, Ensaio: Avaliação e Políticas Públicas em Educação, 25 (96), 721-742. http://dx.doi.org/10.1590/s010440362017002500809

Rodríguez, A. (2015). Un currículo para múltiples adaptaciones. Madrid: EOS.

Salvador, F. (2001). Enciclopedia psicopedagógica de necesidades educativas especiales. Málaga: Aljibe.

Simón. C., Echeita, G., Sandoval, M., Moreno, A., Márquez, C., Fernández, M.L. y Pérez, E. (2016). De las adaptaciones curriculares al diseño universal para el aprendizaje y la instrucción: un cambio de perspectiva. Actas del Congreso Accesibilidad, Ajustes y Apoyos. Proyecto "Madrid sin Barreras". Madrid. 
UNESCO (1994). Declaración de

Salamanca y marco de acción sobre Necesidades Educativas Especiales. Paris: UNESCO.

UNESCO (2008). La educación inclusiva. El camino hacia el futuro. París: UNESCO. http://www.ibe.unesco.org/filead min/user_upload/Policy_Dialogu e/48th_ICE/C ONFINTED_483_Spanish.pdf UNICEF (2013). Estado Mundial de la Infancia. 2013. N

Verdugo, M.A. y Shalock, R. (Coords.) (2014). Discapacidad e inclusión. Manual para la docencia. Salamanca: Amaru.

Warnock, M. (1978). Special educational needs: report of the Committee of Enquiry into the Education of Handicapped Children and Young People. London: Her Majesty's Stationary Office. 